\title{
Prognostic factors in Epstein-Barr virus-associated stage I-III gastric carcinoma: Implications for a unique type of carcinogenesis
}

\author{
SHIH-CHIANG HUANG ${ }^{1}$, KWAI-FONG NG ${ }^{1}$, KUANG-HUA CHEN $^{1}$, JUN-TE HSU ${ }^{2}$, \\ KENG-HAO LIU ${ }^{2}$, TA-SEN YEH ${ }^{2}$ and TSE-CHING CHEN ${ }^{1}$ \\ Departments of ${ }^{1}$ Pathology and ${ }^{2}$ Surgery, Chang Gung Memorial Hospital, Chang Gung University, \\ College of Medicine, Guishan, Taoyuan 333, Taiwan, R.O.C. \\ Received February 20, 2014; Accepted May 9, 2014
}

DOI: $10.3892 /$ or.2014.3234

\begin{abstract}
Epstein-Barr virus-associated gastric carcinoma (EBVaGC) has distinct clinicopathological features. However, the prognostic factors remain unclear, particularly in UICC/AJCC stage I-III cancer. We retrospectively enrolled 1,020 patients with stage I-III gastric cancer that received radical gastrectomy with lymphadenectomy. Formalin-fixed, paraffin-embedded surgical specimens were retrieved to construct tissue microarrays. EBV positivity was identified by in situ hybridization with EBV-encoded small RNA, and the histological classification was reviewed. Fifty-two cases of EBVaGC were identified, exhibiting a male predominance $(\mathrm{p}=0.003)$, a higher prevalence in stump cancer $(\mathrm{p}<0.001)$, and poorly differentiated carcinoma $(\mathrm{p}=0.010)$ compared with the controls. The survival analysis revealed no difference in survival between the EBVaGC cases and the EBV-negative cases $(p=0.977)$. The multivariate analysis showed that EBVaGC cases with a tumor size $>5 \mathrm{~cm}$, non-lymphoepithelioma-like carcinoma (LELC), or a lymph node ratio $>0.15$ had a worse overall survival (hazard ratio 2.884, 12.178 and $19.352 ; \mathrm{p}=0.027,0.005$ and $<0.0001$, respectively). The depth of tumor invasion and the number of lymph node metastases did not reach statistical significance $(\mathrm{p}=0.834$ and 0.833 , respectively). These prognostic factors, tumor size, LELC classification and lymph node ratio, may reflect a unique type of carcinogenesis of EBVaGC and may be considered when selecting high-risk patients for adjuvant treatment.
\end{abstract}

Correspondence to: Professor Ta-Sen Yeh, Department of Surgery, Chang Gung Memorial Hospital, No. 5 Fuxing Street, Guishan Township, Taoyuan County 333, Taiwan, R.O.C.

E-mail: tsy471027@adm.cgmh.org.tw

Professor Tse-Ching Chen, Department of Pathology, Chang Gung Memorial Hospital, No. 5 Fuxing Street, Guishan Township, Taoyuan County 333, Taiwan, R.O.C.

E-mail: ctc323@mail.cgu.edu.tw

Key words: gastric cancer, Epstein-Barr virus, lymphoepitheliomalike carcinoma, lymph node ratio, tumor size

\section{Introduction}

Epstein-Barr virus (EBV) is a ubiquitous $\gamma$-herpes virus that maintains a life-long latent infection in B lymphocytes in over $90 \%$ of adults following salivary transmission during childhood or adolescence (1). Since its discovery in tumor cells of Burkitt's lymphoma 40 years ago, EBV has been associated with various types of cancers, including lymphoid neoplasms, nasopharyngeal and gastric epithelial malignancies, and a subset of mesenchymal tumors $(1,2)$. EBV-associated gastric carcinoma (EBVaGC) accounts for $\sim 10 \%$ of gastric cancer cases (3-5). Gastric cancer has the fourth highest incidence of all types of cancers worldwide, and the burden of EBVaGC is estimated at 75,000-90,000 new cases annually, representing the largest subpopulation among EBV-related tumors $(1,6)$.

From the viewpoint of the clinical distribution, EBVaGC presents the distinct characteristics of a male preponderance, proximal location and high incidence in stump cancer $(3-5,7,8)$. In fact, EBVaGC is a heterogeneous histological group consisting of lymphoepithelioma-like carcinoma (LELC) and conventional adenocarcinoma $(3,4,7)$. More than $80 \%$ of LELC cases are associated with EBV infection, while only $5-10 \%$ of ordinary adenocarcinoma cases are positive for EBV infection. The LELC histotype has been demonstrated to present a significantly favorable prognosis as a result of extensive infiltration of $\mathrm{CD}^{+} \mathrm{T}$ cells and mature dendritic cells within these tumors (9-11). Nevertheless, controversy still exists regarding the prognostic significance of EBV infection itself. Several studies reported a better prognosis in EBVaGC, but a favorable outcome may not be observed after adjusting for other clinicopathological features $(11,12)$. Other researchers have failed to show that EBVaGC differs from EBV-negative gastric cancer in terms of survival $(8,13$ 15). There is a paucity of studies regarding the impact of clinicopathological factors on survival in EBVaGC. A recent study showed that advanced stage and histological classification were meaningful indicators of survival (11).

Since stage I-III gastric cancer patients comprise a population with diverse outcomes, there is considerable interest in understanding the predictors of this cancer's behavior beyond 
the Union for International Cancer Control/American Joint Committee on Cancer (UICC/AJCC) stage. These factors, including the tumor size and lymph node (LN) ratio, defined as the ratio of metastatic to retrieved lymph nodes, have not yet been examined specifically in EBVaGC. Therefore, the present study was conducted to investigate the prognostic significance of the relevant clinicopathological parameters in stage I-III $\mathrm{EBVaGC}$ and to determine the predictive factors that would aid in the identification of high-risk patients who may benefit from adjuvant therapy.

\section{Materials and methods}

Case selection. We retrospectively enrolled gastric cancer cases according to the following criteria: radical resection with lymph node dissection and final pathologic stage I to III according to the 2010 UICC/AJCC staging system. From January 1999 to December 2006, 1,020 consecutive surgically resected specimens of gastric carcinoma were retrieved from the archives of the Department of Pathology at Chang Chung Memorial Hospital, Linkou, Taiwan. Clinical information concerning the patient characteristics and outcome was collected from the medical records. The survival period was traced until December 31, 2012. The study was approved by the institutional review board at our hospital.

Tissue microarrays and EBV-encoded small RNA in situ hybridization. The formalin-fixed and paraffin-embedded tissue samples were arrayed using an automated tissue-arraying machine (Beecher ATA-27; Beecher Instruments, Sun Prairie, WI, USA). All hematoxylin and eosin-stained slides were reviewed to choose representative tumor areas. Three $1.0-\mathrm{mm}$ tissue cores were taken from tissue blocks and transferred to receipt blocks. The tissue microarrays were used for EBV-encoded small RNA (EBER) in situ hybridization.

EBER in situ hybridization was performed on tissue microarray slides using the EBV Probe ISH kit from Leica Microsystems in an automated immunostaining machine (BOND-MAX ${ }^{\mathrm{TM}}$; Leica, Wetzlar, Germany). The procedures were conducted according to the manufacturer's instructions. Only cases with universal and strong nuclear staining within almost all tumor cells were interpreted as EBV-positive.

Pathological analysis. More than two hematoxylin and eosinstained slides of each EBVaGC were reviewed blindly by two pathologists (Dr S.C. Huang and Dr T.C. Chen) to determine the tumor type. We subclassified the EBVaGC specimens into LELC, tubular adenocarcinoma, poorly cohesive adenocarcinoma and mucinous adenocarcinoma. LELC was defined by the Watanabe's and Shibata's criteria: well circumscribed, undifferentiated carcinoma, non-desmoplastic stroma with dense lymphoplasmacytic infiltration $(10,16)$. Tubular adenocarcinoma, poorly cohesive adenocarcinoma, and mucinous adenocarcinoma were defined as tumors with $>50 \%$ of the tumor cells growing in a tubular pattern, poor cohesion and mucin pools, respectively, according to WHO classification (17). Mixed carcinoma was defined as a mixture of tubular adenocarcinoma and poorly cohesive carcinoma if either component accounted for $>10 \%$ of the tumor.
Immunohistochemistry of HER2. We used a HER2 monoclonal antibody (A485, 1:200; Dako, Carpinteria, CA, USA) for immunostaining. Tissue sections of EBVaGC were prepared at a thickness of 3- $\mu \mathrm{m}$ and deparaffinized in xylene and rehydrated in a graded ethanol series. The slides were submitted to antigen retrieval, antibody incubation and chromogen counterstaining in an automated immunostainer (BOND-MAX ${ }^{\mathrm{TM}}$ ). Optimal positive and negative controls were also performed at the same time. Two experienced pathologists interpreted the HER2 status according to the recommendation for gastric cancer (18).

Statistical analysis. Statistical analysis was performed on an SPSS platform (version 17; SPSS, Chicago, IL, USA). The associations between the clinicopathological characteristics and the EBV status were evaluated by independent t-test, Pearson's $\chi^{2}$ or Fisher exact test, according to the variable type. A multivariate logistic regression model was applied to the variables with a p-value $<0.05$ in the univariate analysis. For the comparison of LELC and non-LELC EBVaGC, the Mann-Whitney U test was employed for some continuous variables, including age, size and $\mathrm{LN}$ ratio. The overall survival was measured from the date of surgery to the date of death. Kaplan-Meier estimate was performed to calculate the overall survival, and the statistical significance of different variables was examined by the log-rank test. The Cox proportional hazard regression model was undertaken to determine the independent prognostic factors. Two-sided p-values were calculated, and $\mathrm{p}<0.05$ was considered to be significant for all statistical analyses.

\section{Results}

Patient characteristics and pathological findings. A total of 52 EBVaGCs $(5.1 \%)$ were identified by EBER in situ hybridization from 1,020 stage I-III gastric cancers. The clinical and pathologic features of the cases of EBVaGC and EBV-negative gastric cancer are summarized in Table I. The EBVaGC cases included 43 men and 9 women with a mean age of 64.83 years. The multivariate logistic regression analysis demonstrated that $\mathrm{EBVaGC}$ had a male predominance [odds ratio (OR) 3.120 , 95\% confidence interval (CI) 1.473-6.612, $\mathrm{p}=0.003$ ] and a higher incidence in stump cancers (OR 5.957, 95\% CI 2.423-14.648, $\mathrm{p}<0.0001)$ and poorly differentiated adenocarcinoma (OR 2.494, 95\% CI 1.249-4.981, $\mathrm{p}=0.010$ ) in comparison to EBV-negative gastric cancer. The EBVaGCs tended to occur more frequently in the proximal and middle portion than EBV-negative gastric cancer (57.7 vs. 32.0\%), although the multivariate analysis failed due to the occurrence of nonconvergence in the logistic regression models. No significant difference was found in regards to patient age, Lauren's classification, depth of invasion, metastatic node number, UICC/ AJCC stage, lymphovascular permeation, perineural invasion and Helicobacter infection status between the EBVaGCs and EBV-negative gastric cancer. The tumor size and LN ratio were also not significantly different.

The 52 cases of EBVaGC were further subclassified into 19 LELC cases (36.5\%) and 33 non-LELC cases (63.5\%) (Fig. 1). The 33 non-LELC EBVaGC cases consisted of 29 cases of tubular adenocarcinoma $(87.9 \%), 1$ case of poorly cohesive carcinoma $(3.0 \%), 1$ case of mucinous adenocarcinoma $(3.0 \%)$ 
Table I. Clinicopathological findings of stage I-III gastric cancer patients classified by EBV status.

\begin{tabular}{|c|c|c|c|c|c|c|}
\hline \multirow[b]{3}{*}{ Parameters } & \multicolumn{2}{|c|}{ EBV status } & \multirow{3}{*}{$\begin{array}{c}\begin{array}{c}\text { Univariate } \\
\text { analysis }\end{array} \\
\text { P-value }\end{array}$} & \multirow{2}{*}{\multicolumn{3}{|c|}{$\begin{array}{l}\text { Multivariate } \\
\text { analysis }\end{array}$}} \\
\hline & \multirow{2}{*}{$\begin{array}{l}\text { Negative } \\
(\mathrm{n}=968)\end{array}$} & \multirow{2}{*}{$\begin{array}{c}\text { Positive } \\
(\mathrm{n}=52)\end{array}$} & & & & \\
\hline & & & & Odds ratio & $95 \% \mathrm{CI}$ & P-value \\
\hline Age (years), mean \pm SD & $63.65 \pm 13.51$ & $64.83 \pm 11.02$ & 0.536 & & & \\
\hline Gender & & & 0.001 & & & \\
\hline Male & $567(58.6)$ & $43(82.7)$ & & 3.120 & $1.473-6.612$ & 0.003 \\
\hline Female & $401(41.4)$ & $9(17.3)$ & & 1 & & \\
\hline Stump cancer & & & $<0.0001$ & & & \\
\hline Yes & $21(2.2)$ & $10(19.2)$ & & 5.957 & $2.423-14.648$ & $<0.001$ \\
\hline No & $947(97.8)$ & $42(80.8)$ & & 1 & & \\
\hline Localization $^{\text {a }}$ & & & $<0.001$ & & & \\
\hline Upper & $149(15.4)$ & $20(38.5)$ & & & & \\
\hline Middle & $161(16.6)$ & $10(19.2)$ & & & & \\
\hline Lower & $620(64.0)$ & $21(40.4)$ & & & & \\
\hline Diffuse & $38 \quad(3.9)$ & $1(1.9)$ & & & & \\
\hline Tumor size, mean $\pm \mathrm{SD}(\mathrm{cm})$ & $4.43 \pm 3.17$ & $4.44 \pm 2.36$ & 0.966 & & & \\
\hline Differentiation & & & 0.010 & & & \\
\hline Well/moderate & $397(41.0)$ & $12(23.1)$ & & 1 & & \\
\hline Poor & $571(59.0)$ & $40(76.9)$ & & 2.494 & $1.249-4.981$ & 0.010 \\
\hline Lauren's classification & & & 0.120 & & & \\
\hline Intestinal & $489(50.5)$ & $25(48.1)$ & & & & \\
\hline Diffuse & $365(37.7)$ & $16(30.8)$ & & & & \\
\hline Mixed & $114(11.8)$ & $11(21.2)$ & & & & \\
\hline Depth of invasion & & & 0.039 & & & \\
\hline $\mathrm{T} 1 / \mathrm{T} 2$ & $360(37.2)$ & $12(23.1)$ & & 1 & & \\
\hline $\mathrm{T} 3 / \mathrm{T} 4$ & $608(62.8)$ & $40(76.9)$ & & 1.233 & $0.604-2.520$ & 0.565 \\
\hline Nodal status & & & 0.753 & & & \\
\hline No & $384(39.7)$ & $17(32.7)$ & & & & \\
\hline $\mathrm{N} 1$ & $133(13.7)$ & $7(13.5)$ & & & & \\
\hline $\mathrm{N} 2$ & $154(15.9)$ & $10(19.2)$ & & & & \\
\hline N3 & $297(30.7)$ & $18(34.6)$ & & & & \\
\hline Stage & & & 0.179 & & & \\
\hline I & $283(29.2)$ & $9(17.3)$ & & & & \\
\hline II & $177(18.3)$ & $11(21.2)$ & & & & \\
\hline III & $508(52.5)$ & $32(61.5)$ & & & & \\
\hline $\mathrm{LN}$ ratio, mean $\pm \mathrm{SD}$ & $0.21 \pm 0.27$ & $0.19 \pm 0.21$ & 0.702 & & & \\
\hline Lymphatic invasion ${ }^{\mathrm{b}}$ & & & 0.204 & & & \\
\hline No & $475(49.4)$ & $21(40.4)$ & & & & \\
\hline Yes & $486(50.6)$ & $31(59.6)$ & & & & \\
\hline Vascular invasion ${ }^{\mathrm{b}}$ & & & 0.863 & & & \\
\hline No & $838(87.7)$ & $46(88.5)$ & & & & \\
\hline Yes & $118(12.3)$ & $6(11.5)$ & & & & \\
\hline Perineural invasion ${ }^{\mathrm{b}}$ & & & 0.892 & & & \\
\hline No & $505(52.9)$ & $28(53.8)$ & & & & \\
\hline Yes & $450(47.1)$ & $24(46.2)$ & & & & \\
\hline HP infection & & & 0.462 & & & \\
\hline No & $771(80.5)$ & $44(84.6)$ & & & & \\
\hline Yes & $187(19.5)$ & $8(15.4)$ & & & & \\
\hline
\end{tabular}

Data are numbers with percentages in parentheses, unless otherwise stated. EBV, Epstein-Barr virus; SD, standard deviation; LN ratio, lymph

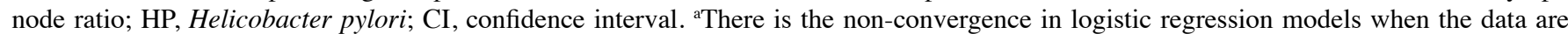

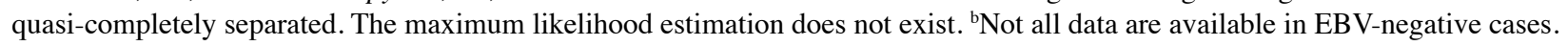


Table II. Clinicopathological findings of stage I-III EBV-associated gastric cancer patients classified by tumor histology.

\begin{tabular}{|c|c|c|c|}
\hline \multirow[b]{2}{*}{ Parameters } & \multicolumn{2}{|c|}{ Tumor type } & \multirow[b]{2}{*}{ P-value } \\
\hline & $\begin{array}{l}\text { LELC } \\
(\mathrm{n}=18)\end{array}$ & $\begin{array}{l}\text { Non-LELC } \\
\quad(\mathrm{n}=33)\end{array}$ & \\
\hline Age (years), median (range) & $62(44-81)$ & $70(39-80)$ & 0.253 \\
\hline Gender & & & 0.703 \\
\hline Male & $14(77.8)$ & $28(984.8)$ & \\
\hline Female & $4(22.2)$ & $5 \quad(15.2)$ & \\
\hline Stump cancer & & & 0.727 \\
\hline Yes & $4(22.2)$ & $6(18.2)$ & \\
\hline No & $14(77.8)$ & $27(81.8)$ & \\
\hline Localization & & & 0.322 \\
\hline Upper & $5(27.8)$ & $15(45.5)$ & \\
\hline Middle & $3(16.7)$ & $7(21.2)$ & \\
\hline Lower & $10(55.6)$ & $10(30.3)$ & \\
\hline Diffuse & 0 & $1 \quad(3.0)$ & \\
\hline Tumor size $(\mathrm{cm})$, median (range) & $3.8(1.0-6.0)$ & $4.5(0.8-14.0)$ & 0.161 \\
\hline Differentiation & & & 0.004 \\
\hline Well/moderate & 0 & $12(36.4)$ & \\
\hline Poor & $18(100.0)$ & 21 (63.6) & \\
\hline Lauren's classification & & & 0.106 \\
\hline Intestinal & $5(27.8)$ & $19(57.6)$ & \\
\hline Diffuse & $7(38.9)$ & $9(27.3)$ & \\
\hline Mixed & $6(33.3)$ & $5(15.2)$ & \\
\hline Depth of invasion & & & 0.304 \\
\hline $\mathrm{T} 1 / \mathrm{T} 2$ & $6(33.3)$ & $6(18.2)$ & \\
\hline $\mathrm{T} 3 / \mathrm{T} 4$ & $12(66.7)$ & $27(81.8)$ & \\
\hline Nodal status & & & 0.282 \\
\hline N0 & $9(50.0)$ & $8(24.2)$ & \\
\hline N1 & $2(11.1)$ & $5(15.2)$ & \\
\hline $\mathrm{N} 2$ & $3(16.7)$ & $6(18.2)$ & \\
\hline N3 & $4(22.2)$ & $14(42.4)$ & \\
\hline Stage & & & 0.189 \\
\hline $\mathrm{I}$ & $5(27.8)$ & $4(12.1)$ & \\
\hline II & $5(27.8)$ & $6(18.2)$ & \\
\hline III & $8(44.4)$ & $23(69.7)$ & \\
\hline LN ratio, median (range) & $0.03(0-0.57)$ & $0.19(0-0.81)$ & 0.116 \\
\hline Lymphatic invasion & & & 0.244 \\
\hline No & $9(50.0)$ & $11(33.3)$ & \\
\hline Yes & $9(50.0)$ & $22(66.7)$ & \\
\hline Vascular invasion & & & 0.078 \\
\hline No & $18(100.0)$ & 27 (81.8) & \\
\hline Yes & 0 & $6(18.2)$ & \\
\hline Perineural invasion & & & 0.147 \\
\hline No & $12(66.7)$ & $15(45.5)$ & \\
\hline Yes & $6(33.3)$ & $18(54.5)$ & \\
\hline HP infection & & & 0.686 \\
\hline No & $15(83.3)$ & $29(87.9)$ & \\
\hline Yes & $3(16.7)$ & $4(12.1)$ & \\
\hline
\end{tabular}

Figures are numbers with percentages in parentheses, unless otherwise stated. EBV, Epstein-Barr virus; LELC, lymphoepithelioma-like carcinoma; LN ratio, lymph node ratio; HP, Helicobacter pylori. 

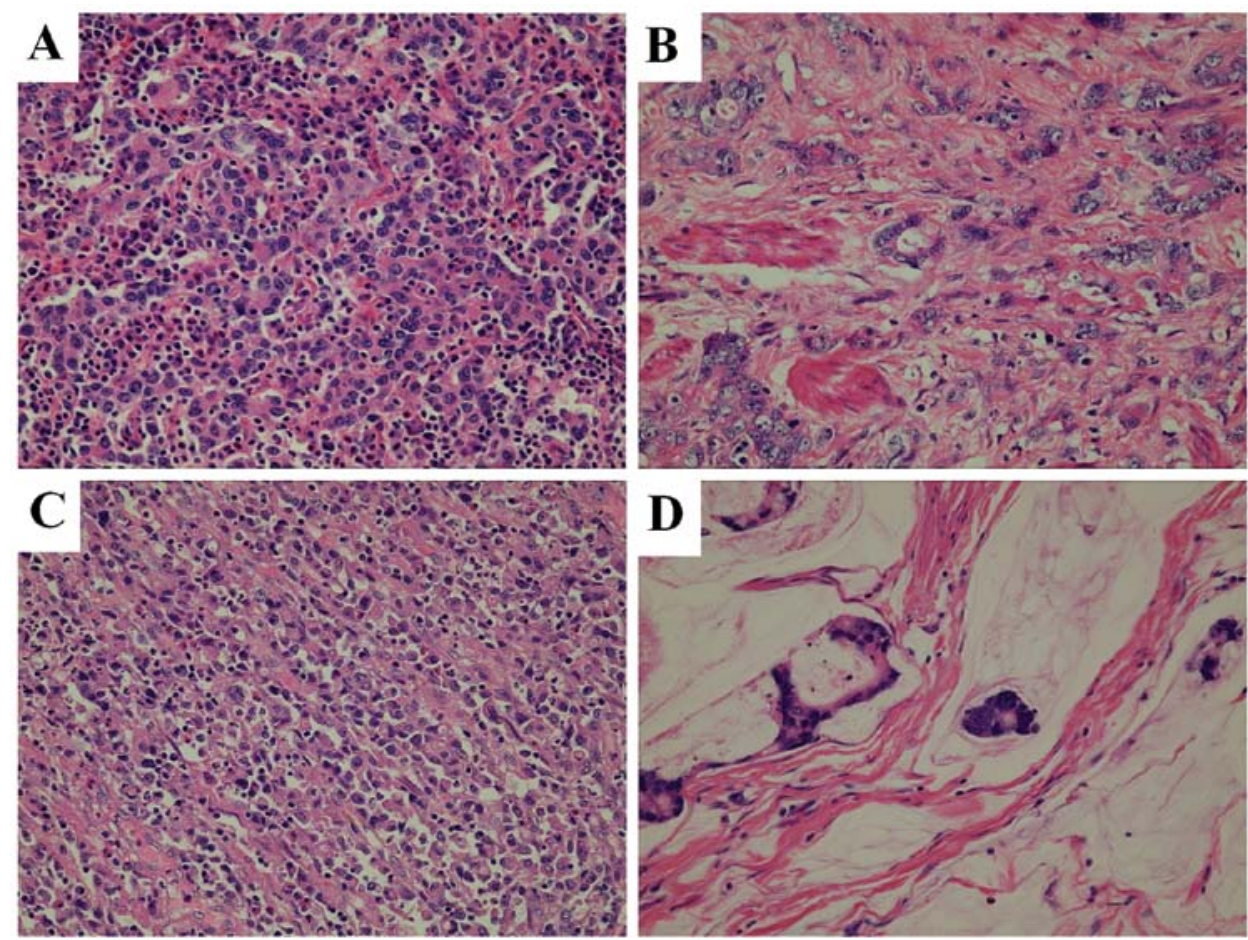

Figure 1. Histological classification of Epstein-Barr virus-associated gastric cancer: (A) lymphoepithelioma-like carcinoma, (B) tubular adenocarcinoma, (C) poorly cohesive carcinoma and (D) mucinous adenocarcinoma (magnification, $\mathrm{x} 400$ ).

and 2 cases of mixed carcinoma (6.1\%). After excluding 1 case that suffered from surgical mortality, the clinicopathological parameters of LELC and non-LELC in the 51 cases of EBVaGC were not significantly different, with the exception of tumor differentiation $(\mathrm{p}=0.004)$ (Table II).

HER2 immunohistochemistry. Only one case of the 52 EBVaGCs (1.9\%) demonstrated HER2 overexpression (strong intensity, score $3^{+}$).

Survival analysis. According to the available survival data, the mean survival duration for the 51 cases of EBVaGC and the 943 cases of EBV-negative gastric cancer was 87.74 (95\% CI 70.37-105.11) and 88.1 (95\% CI 84.0992.12) months, respectively. The follow-up duration for the 51 cases of EBVaGC ranged from 1.2 to 166 months (median 45.04 months). EBV infection itself was not a prognostic factor in stage I-III gastric cancer $(\mathrm{p}=0.977)$ (Fig. 2). According to the survival analysis of EBVaGC, the log-rank analysis revealed that tumor location, tumor size $>5 \mathrm{~cm}$, depth of tumor invasion, number of lymph node metastasis, LN ratio, lymphovascular invasion, perineural invasion and LELC subtype reached statistical significance as prognostic factors (Table III). In the Cox proportional hazard model, tumor size $>5 \mathrm{~cm}$ [hazard ratio (HR) $2.884,95 \% \mathrm{CI}$ 1.129-7.365, $\mathrm{p}=0.027], \mathrm{LN}$ ratio $>0.15(\mathrm{HR} 19.352,95 \% \mathrm{CI}$ 4.383-85.441, $\mathrm{p}<0.0001$ ), and non-LELC subtypes (HR $12.178,95 \%$ CI $2.135-69.474, \mathrm{p}=0.005$ ) had an unfavorable effect on survival. However, the depth of tumor invasion and nodal status lost their statistical significance $(\mathrm{p}=0.834$ and 0.844 , respectively). Tumor location was excluded due to the occurrence of the monotone likelihood, and the maximum

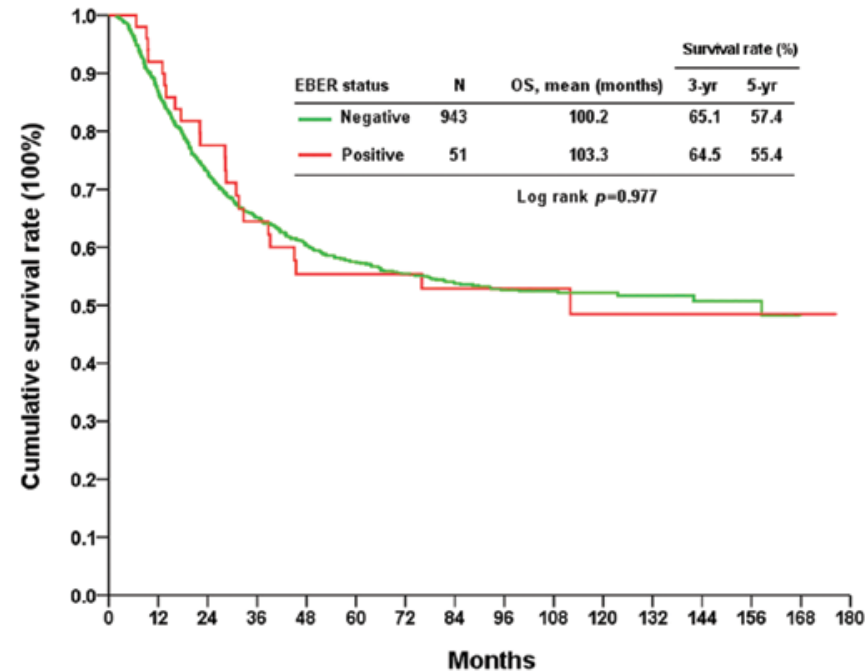

Figure 2. Comparison of Kaplan-Meier estimated survival curves of gastric cancer according to the results of EBV-encoded small RNA (EBER) in situ hybridization.

likelihood estimation did not exist. The median survival for cases with a tumor size $>5 \mathrm{~cm}$ was 22.1 months with a 5 -year survival rate of $13.3 \%$ compared to $74.9 \%$ in tumors with a size $\leq 5 \mathrm{~cm}$ (Fig. 3A). LELC EBVaGC had a 5-year survival rate of $87.5 \%$, which was superior to the non-LELC cases with a 5-year survival rate of $38.4 \%$ and a median survival of 32.7 months (Fig. 3B). Cases with an LN ratio $>0.15$ had a median survival of 28.3 months and a $13.4 \% 5$-year survival rate, whereas cases with an LN ratio $\leq 0.15$ had a 96.0\% 5-year survival rate (Fig. 3C). 
Table III. Survival analysis of patients with stage I-III EBV-associated gastric cancer.

\begin{tabular}{|c|c|c|c|c|c|c|}
\hline \multirow{2}{*}{$\begin{array}{l}\text { Clinicopathological } \\
\text { factors }\end{array}$} & \multicolumn{3}{|c|}{ Univariate analysis } & \multicolumn{3}{|c|}{ Multivariate analysis } \\
\hline & (months) & $95 \% \mathrm{CI}$ & P-value & Hazard ratio & $95 \% \mathrm{CI}$ & P-value \\
\hline Age (years) & & & 0.116 & & & \\
\hline$\leq 65(\mathrm{n}=25)$ & 120.81 & $91.59-150.03$ & & & & \\
\hline$>65(n=26)$ & 79.73 & $51.57-107.90$ & & & & \\
\hline Gender & & & 0.859 & & & \\
\hline Male $(n=42)$ & 101.62 & $77.56-125.68$ & & & & \\
\hline Female $(n=9)$ & 80.24 & $49.32-111.15$ & & & & \\
\hline Location $^{\text {a }}$ & & & $<0.0001$ & & & \\
\hline Upper $(n=20)$ & 79.68 & 49.76-109.61 & & & & \\
\hline Middle $(\mathrm{n}=10)$ & 102.77 & $60.51-145.02$ & & & & \\
\hline Lower $(n=20)$ & 119.82 & $86.44-153.21$ & & & & \\
\hline Diffuse $(n=1)$ & 6.67 & $6.67-6.67$ & & & & \\
\hline Tumor size (cm) & & & $<0.0001$ & & & \\
\hline$\leq 5(\mathrm{n}=35)$ & 135.28 & $112.41-158.14$ & & 1 & & \\
\hline$>5(\mathrm{n}=16)$ & 30.58 & $17.45-43.71$ & & 2.884 & $1.129-7.365$ & 0.027 \\
\hline Differentiation & & & 0.892 & & & \\
\hline Well/moderate $(\mathrm{n}=12)$ & 86.48 & $51.27-121.69$ & & & & \\
\hline Poor $(n=39)$ & 105.19 & $80.19-130.20$ & & & & \\
\hline Histological classification & & & $<0.0001$ & & & \\
\hline $\operatorname{LELC~}(n=18)$ & 158.97 & $136.27-181.68$ & & 1 & & \\
\hline Non-LELC $(\mathrm{n}=33)$ & 67.04 & $44.61-89.47$ & & 12.178 & $2.135-69.474$ & 0.005 \\
\hline Depth of invasion & & & 0.032 & & & 0.834 \\
\hline $\mathrm{T} 1 / \mathrm{T} 2(\mathrm{n}=12)$ & 145.23 & 118.37-172.09 & & & & \\
\hline T3/T4 $(\mathrm{n}=39)$ & 90.10 & $65.54-114.65$ & & & & \\
\hline Nodal status & & & $<0.0001$ & & & 0.844 \\
\hline N0 $(n=17)$ & 160.30 & $145.92-174.69$ & & & & \\
\hline $\mathrm{N} 1(\mathrm{n}=7)$ & 116.73 & $86.43-147.03$ & & & & \\
\hline $\mathrm{N} 2(\mathrm{n}=9)$ & 79.00 & $27.26-131.93$ & & & & \\
\hline N3 (n=18) & 37.42 & $20.87-53.98$ & & & & \\
\hline $\mathrm{LN}$ ratio & & & $<0.0001$ & & & \\
\hline$\leq 0.15(\mathrm{n}=27)$ & 151.83 & 134.94-168.72 & & 1 & & \\
\hline$>0.15(\mathrm{n}=24)$ & 44.72 & $22.92-66.51$ & & 19.352 & $4.383-85.441$ & $<0.0001$ \\
\hline Lymphatic invasion & & & 0.001 & & & 0.699 \\
\hline No $(n=20)$ & 146.21 & 123.60-168.81 & & & & \\
\hline Yes $(n=31)$ & 72.10 & $46.34-97.87$ & & & & \\
\hline Vascular invasion & & & 0.003 & & & 0.935 \\
\hline No $(n=45)$ & 114.57 & $91.82-137.31$ & & & & \\
\hline Yes $(n=6)$ & 36.97 & $6.17-67.77$ & & & & \\
\hline Perineural invasion & & & 0.002 & & & 0.464 \\
\hline No $(n=27)$ & 132.40 & $106.92-157.88$ & & & & \\
\hline Yes $(n=24)$ & 63.63 & $36.68-90.58$ & & & & \\
\hline HP infection & & & 0.846 & & & \\
\hline No $(n=44)$ & 101.76 & 79.04-124.47 & & & & \\
\hline Yes $(n=7)$ & 104.29 & $42.14-166.44$ & & & & \\
\hline
\end{tabular}

EBV, Epstein-Barr virus; LELC, lymphoepithelioma-like carcinoma; LN ratio, lymph node ratio; HP, Helicobacter pylori; CI, confidence

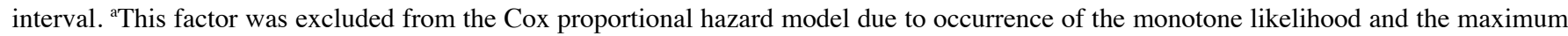
likelihood estimation did not exist. 


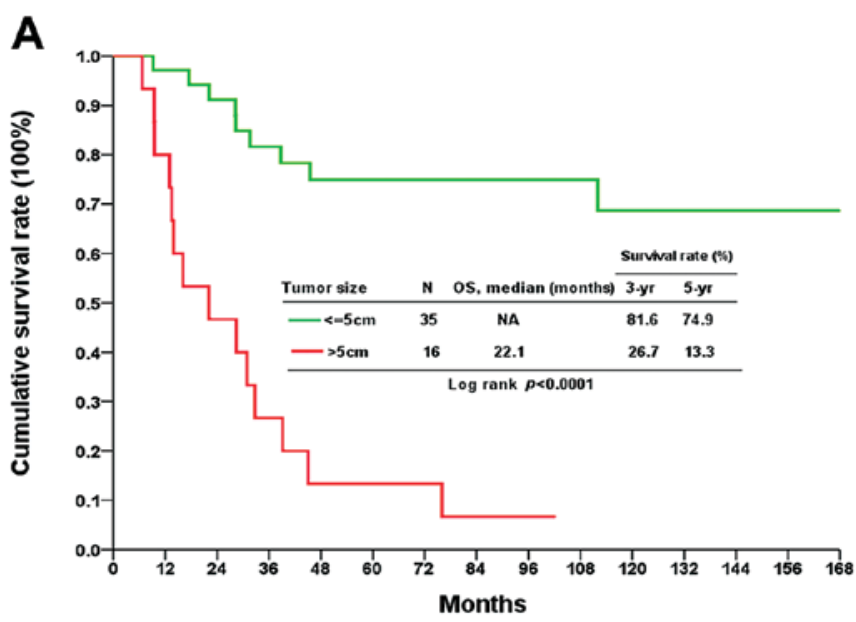

B
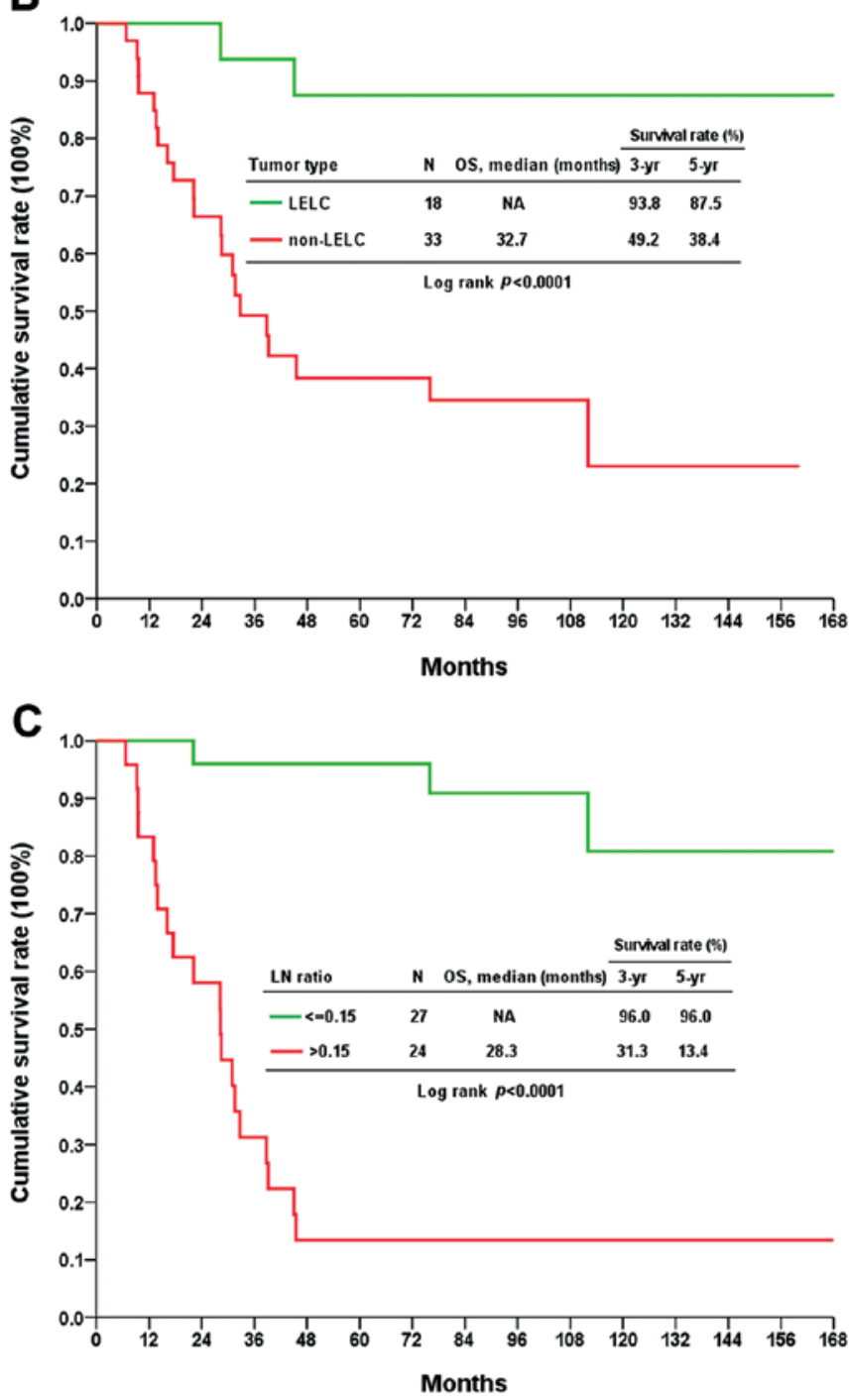

Figure 3. Overall survival analysis of 51 Epstein-Barr virus-associated gastric cancer cases according to different prognostic factors: (A) tumor size, (B) histological lymphoepithelioma-like carcinoma (LELC) classification and $(\mathrm{C})$ lymph node ratio (LN ratio).

\section{Discussion}

In the present study, EBVaGC constituted $5.1 \%$ of the cases of stage I-III gastric cancer and was associated with demographic features that have been described in previous studies. According to a large-scale meta-analysis of 70 studies including 15,952 cases of gastric cancer, EBVaGC had a prevalence estimate of $8.7 \%$ (95\% CI $7.5-10.0 \%$, range $1.33-19.9 \%$ ) (5). The metaanalysis revealed that EBVaGC presented a 2-fold higher incidence in males $(11.1 \%)$ than in females and occurred twice as often in the gastric cardia or corpus than in the antrum (13.6 or 13.1 vs. $5.2 \%$ ) compared to EBV-negative gastric cancers. Stump cancer had a 4-fold higher incidence of EBV positivity (35.1\%). The above results are similar to the finding of our study, which revealed a male preponderance and a higher incidence in stump cancer. Regarding the location in the stomach, EBVaGC seems to occur more frequently in the upper third and less frequently in the lower third than EBV-negative gastric cancer. No significant difference was observed in prevalence between the intestinal, diffuse and mixed tumor types. In addition, the present study revealed that poorly differentiated carcinoma has a higher likeliness of EBV positivity. Taiwan is an endemic area for nasopharyngeal cancer, which is also associated with EBV infection. The data gathered at our institute indicated that EBVaGC was a rare $(1 / 6,16.6 \%)$ event of secondary cancer in patients with nasopharyngeal cancer in Taiwan (19). Furthermore, the incidence of EBVaGC is similar worldwide, with $8.3 \%$ in Asia, 9.2\% in Europe and 9.9\% in America (5). EBV-associated gastric cancer and nasopharyngeal cancer express latency I and latency II protein, respectively $(1,2)$. EBVaGC may be a universal and distinct variant of gastric cancer with characteristic clinical features that are independent from nasopharyngeal cancer.

In addition, our results showed that EBV infection itself seems not to be a prognostic factor, and risk stratification by tumor size, histological classification, and LN ratio may help identify high-risk patients. According to the literature, a favorable outcome was observed only in those cases categorized as LELC, and EBV infection itself was not associated with any survival advantage (10,11,13-15). Our results also support this prevailing opinion. LELC-type EBVaGC presents a striking benefit for both the 3- and 5-year survival rate. This unique histological subtype is distinguished by the presence of a non-desmoplastic stroma infiltrated with an abundance of lymphocytes and plasma cells $(10,16)$. These extensive intratumoral inflammatory components are composed of activated cytotoxic $\mathrm{T}$, nature killer and mature dendritic cells $(9,15,20)$. The $\mathrm{T}$ cell infiltration has been correlated to intratumoral FoxP3-positive regulatory T cells (21). Some authors hypothesized that the intratumoral inflammatory reaction represents an effective host immune reaction against tumor cells $(9,20,22)$. In colon, breast and lung malignancies, tumor-infiltrating lymphocytes also express a cytotoxic T-cell phenotype and are related to a survival advantage (22).

Likewise, the intratumoral inflammation pattern and intensity have been reported to influence the survival of EBVaGC patients $(9,11,22)$. Song et al purported that a typical Crohn's disease-like lymphocytic reaction may share a similar morphology with LELC on the basis of the similar survival benefit in both groups (11). Grogg et al found that increased lymphocyte infiltration of the tumor indicated a better prognosis as EBVaGC had a higher lymphocyte count (450/10 HPF) than EBV-negative gastric cancer (21/10 HPF) (22). The amount of intratumoral $\mathrm{T}$ and dendritic cells was also more plentiful 
in EBVaGC without lymph node metastasis than in EBVaGC with node metastasis (9). Although the underlying mechanism of the antitumor immune reaction is not well elucidated, the accurate classification of EBVaGC into LELC or non-LELC is not only associated with patient prognosis but also implies a different tumor-host interaction.

Tumor size has been regarded as an important prognostic factor due to its close relationship to histological grade, UICC/ AJCC stage, vascular invasion and neural permeation (23). We observed that a tumor size $>5 \mathrm{~cm}$ is an independent parameter of poor prognosis in stage I-III EBVaGC instead of the depth of tumor invasion. EBVaGC frequently grows in ulcerated or saucer-like tumors featured by well-delineated and pushing borders $(7,10,16)$. This macroscopic pattern corresponds to a microscopic expanding front rather than to infiltrative invasion. EBVaGC may follow a different encroachment fashion from EBV-negative gastric cancer. That may be why tumor size is a powerful predictor. Indeed, tumor size is a paramount component for UICC/AJCC tumor stage in some malignancies, including lung and breast cancers (24). For gastric cancer, some authors have found that tumor size is a simple prognostic indicator and could even improve the accuracy of UICC/AJCC staging for gastric cancer $(25,26)$. Due to the heterogeneity of gastric cancer, tumor size may be a significant outcome indicator in some subgroups, such as EBVaGC, as shown by the present study.

The $\mathrm{LN}$ ratio is an emerging parameter that may be more useful than UICC/AJCC lymph node stage due to its consistent prognostic power whenever the type of lymphadenectomy or total number of resected nodes varies (27-30). This study suggests that an $\mathrm{LN}$ ratio $>0.15$ is an independent and powerful predictor that is superior to the UICC/AJCC nodal status in stage I-III EBVaGC. The mean number of harvested lymph nodes from the EBVaGC group in our study was 30.5 with 6 cases having a total of $<15$ lymph nodes $(11.8 \%$ ) (data not shown). An LN ratio $>0.15$ retained its statistical significance in the Cox regression multivariate analysis and had the strongest hazard ratio compared with the other factors. Most previous studies have divided LN ratio into categories corresponding to $0,1-9,10-25$ and $>25 \%$ to stratify patient risk, which is intended to imitate the AJCC/UICC node status $(27,28)$. Our data demonstrated that a simple threshold of 0.15 could achieve considerable discernability for patient outcome. Metastatic foci of EBVaGC in lymph nodes still maintain an EBV genome (9). The occurrence of node metastasis does not signify tumor escape by virus deletion, but it may imply alteration of the tumor to lose the target antigen or to attenuate the immune defense. Indeed, a better prognosis observed in EBVaGC has been attributed to less lymph node involvement (12). The relationship between tumor molecular alteration and lymph node metastasis still requires further study, but the LN ratio may be used as another simple approach to predict this host-tumor interaction and its effect on patient outcome.

EBV is thought to play a critical role, not only in stimulation of an immunologic reaction, but also in gastric carcinogenesis. Through the presentation of an undefined antigen, EBV attracts strikingly numerous lymphocytes and plasma cells that are intimately admixed with tumor cells by upregulation of major histocompatibility complex class II molecules and
IL1- $\beta$ cytokines $(9,31)$. Aside from the differences in protein expression and chromosomal aberrations compared with EBV-negative counterparts, EBVaGC exhibits characteristic molecular features, including a global and non-random $\mathrm{CpG}$ island methylation epigenotype, which is activated by DNMT1 through the phosphorylation of STAT3 from viral LMP2A effect (7,32-36). Considering the unique host-tumor interaction and the molecular alterations detected in EBVaGC, our data suggest that the use of the histological classification, tumor size, and LN ratio could discriminate the prognosis of patients with stage I-III EBVaGC. For therapeutic options that utilize virus-host interactions in EBV-associated tumors, demethylating agents, such as 5-azacytidine, can induce lytic infection of EBV, leading to lysis of the infected tumor cells, by restoring the expression of the BMRF1, BZLF1 and BRLF1 genes after removing promoter methylation $(37,38)$. These novel antitumor drugs may provide a maximal survival benefit to those high-risk patients with a large tumor size, non-LELC tumors or a high LN ratio.

It is important to characterize the tumor pathway and processes for personalized medicine. The present study is one of our serial investigations aimed at the molecular classification of gastric cancers. EBVaGC and HER-2-overexpressing gastric cancers may represent distinct subsets since only one EBVaGC case had HER-2 overexpression. The incidence of $1.9 \%$ was much lower than that of $6.1 \%$ in the general population of gastric cancer, as demonstrated in our previous study (39). In addition, the low incidence of HER2 amplification is also consistent with the results reported by a Korean group $(1 / 123,0.8 \%)(11)$.

In conclusion, we conducted a large-scale study involving 1,020 stage I-III gastric cancer cases from a single institute. We identified 52 cases of EBVaGC. EBVaGC showed a male preponderance and a higher incidence in stump cancer and poorly differentiated carcinoma. The survival analysis suggested that tumor size, LELC classification, and LN ratio were important prognostic factor. These influential factors most likely reflect EBVaGC's unique mode of carcinogenesis and host-tumor interaction and should be considered in the identification of high-risk patients who may benefit from adjuvant regimens or virus-specific treatments.

\section{Acknowledgements}

We thank the Tissue Bank, Chang Gung Memorial Hospital, for excellent tissue processing. This study was supported by a grant from the Department of Health (DOH99-TD-C-111-006), a grant from the National Science Council (101-2320-B-182A008), and a grant from the Chang Gung Memorial Hospital (CMRPG340547, CMRPG3B1192 and CMRPG3A1182).

\section{References}

1. Young LS and Rickinson AB: Epstein-Barr virus: 40 years on. Nat Rev Cancer 4: 757-768, 2004.

2. Deyrup AT: Epstein-Barr virus-associated epithelial and mesenchymal neoplasms. Hum Pathol 39: 473-483, 2008.

3. Shibata D and Weiss LM: Epstein-Barr virus-associated gastric adenocarcinoma. Am J Pathol 140: 769-774, 1992.

4. Tokunaga M, Land CE, Uemura Y, Tokudome T, Tanaka S and Sato E: Epstein-Barr virus in gastric carcinoma. Am J Pathol 143: 1250-1254, 1993. 
5. Murphy G, Pfeiffer R, Camargo MC and Rabkin CS: Metaanalysis shows that prevalence of Epstein-Barr virus-positive gastric cancer differs based on sex and anatomic location. Gastroenterology 137: 824-833, 2009.

6. Parkin DM, Bray F, Ferlay J and Pisani P: Global cancer statistics, 2002. CA Cancer J Clin 55: 74-108, 2005.

7. Fukayama M and Ushiku T: Epstein-Barr virus-associated gastric carcinoma. Pathol Res Pract 207: 529-537, 2011.

8. Tokunaga M, Uemura Y, Tokudome T, et al: Epstein-Barr virus related gastric cancer in Japan: a molecular patho-epidemiological study. Acta Pathol Jpn 43: 574-581, 1993.

9. van Beek J, zur Hausen A, Snel SN, et al: Morphological evidence of an activated cytotoxic T-cell infiltrate in EBV-positive gastric carcinoma preventing lymph node metastases. Am J Surg Pathol 30: 59-65, 2006.

10. Watanabe H, Enjoji $M$ and Imai T: Gastric carcinoma with lymphoid stroma. Its morphologic characteristics and prognostic correlations. Cancer 38: 232-243, 1976

11. Song HJ, Srivastava A, Lee J, et al: Host inflammatory response predicts survival of patients with Epstein-Barr virus-associated gastric carcinoma. Gastroenterology 139: 84-92, 2010.

12. van Beek J,zur Hausen A, Klein Kranenbarg E, et al: EBV-positive gastric adenocarcinomas: a distinct clinicopathologic entity with a low frequency of lymph node involvement. J Clin Oncol 22 : 664-670, 2004

13. Park ES, Do IG, Park CK, et al: Cyclooxygenase-2 is an independent prognostic factor in gastric carcinoma patients receiving adjuvant chemotherapy and is not associated with EBV infection. Clin Cancer Res 15: 291-298, 2009.

14. Chang MS, Lee HS, Kim CW, Kim YI and Kim WH: Clinicopathologic characteristics of Epstein-Barr virus-incorporated gastric cancers in Korea. Pathol Res Pract 197: 395-400, 2001

15. Kijima Y, Ishigami S, Hokita S, Koriyama C, Akiba S, Eizuru Y and Aikou T: The comparison of the prognosis between Epstein-Barr virus (EBV)-positive gastric carcinomas and EBV-negative ones. Cancer Lett 200: 33-40, 2003.

16. Shibata D, Tokunaga M, Uemura Y, Sato E, Tanaka $S$ and Weiss LM: Association of Epstein-Barr virus with undifferentiated gastric carcinomas with intense lymphoid infiltration. Lymphoepithelioma-like carcinoma. Am J Pathol 139: 469-474, 1991.

17. Lauwers GY, Carneiro F, Graham DY, et al: Gastric carcinoma In: WHO Classification of Tumours of the Digestive System. Bosman FT, Carneiro F, Hruban RH, Theise ND (eds). IARC, Lyon, pp48-58, 2010.

18. Hofmann M, Stoss O, Shi D, et al: Assessment of a HER2 scoring system for gastric cancer: results from a validation study. Histopathology 52: 797-805, 2008.

19. Wang CC, Chen ML, Hsu KH, et al: Second malignant tumors in patients with nasopharyngeal carcinoma and their association with Epstein-Barr virus. Int J Cancer 87: 228-231, 2000.

20. Minamoto T, Mai M, Watanabe K, et al: Medullary carcinoma with lymphocytic infiltration of the stomach. Clinicopathologic study of 27 cases and immunohistochemical analysis of the subpopulations of infiltrating lymphocytes in the tumor. Cancer 66: 945-952, 1990.

21. Haas M, Büttner M, Rau TT, Fietkau R, Grabenbauer GG and Distel LV: Inflammation in gastric adenocarcinoma of the cardia: how do EBV infection, Her2 amplification and cancer progression influence tumor-infiltrating lymphocytes? Virchows Arch 458: 403-411, 2011.

22. Grogg KL, Lohse CM, Pankratz VS, Halling KC and Smyrk TC: Lymphocyte-rich gastric cancer: associations with Epstein-Barr virus, microsatellite instability, histology, and survival. Mod Pathol 16: 641-651,2003.
23. Yokota T, Ishiyama S, Saito T, et al: Is tumor size a prognostic indicator for gastric carcinoma? Anticancer Res 22: 3673-3677, 2002.

24. Edge SB Byrd DR, Compton CC, Fritz AG, et al (eds): AJCC Cancer Staging Manual. 7th edition. Springer, New York, NY, pp117-126, 2010.

25. Saito H, Osaki T, Murakami D, et al: Macroscopic tumor size as a simple prognostic indicator in patients with gastric cancer. Am J Surg 192: 296-300, 2006.

26. Lu J, Huang CM, Zheng CH, Li P, Xie JW, Wang JB and Lin JX: Consideration of tumor size improves the accuracy of TNM predictions in patients with gastric cancer after curative gastrectomy. Surg Oncol 22: 167-171, 2013.

27. Xu DZ, Geng QR, Long ZJ, et al: Positive lymph node ratio is an independent prognostic factor in gastric cancer after $\mathrm{d} 2$ resection regardless of the examined number of lymph nodes. Ann Surg Oncol 16: 319-326, 2009.

28. Marchet A, Mocellin S, Ambrosi A, et al: The ratio between metastatic and examined lymph nodes ( $\mathrm{N}$ ratio) is an independent prognostic factor in gastric cancer regardless of the type of lymphadenectomy: results from an Italian multicentric study in 1853 patients. Ann Surg 245: 543-552, 2007.

29. Persiani R, Rausei S, Antonacci V, Biondi A, Casella F, Ciccoritti L and D'Ugo D: Metastatic lymph node ratio: a new staging system for gastric cancer. World J Surg 33: 2106-2111, 2009.

30. Inoue K, Nakane Y,Iiyama $\mathrm{H}$, et al: The superiority of ratio-based lymph node staging in gastric carcinoma. Ann Surg Oncol 9: 27-34, 2002.

31. Chong JM, Sakuma K, Sudo M, et al: Interleukin-1 $\beta$ expression in human gastric carcinoma with Epstein-Barr virus infection. J Virol 76: 6825-6831, 2002.

32. Lee HS, Chang MS, Yang HK, Lee BL and Kim WH Epstein-barr virus-positive gastric carcinoma has a distinct protein expression profile in comparison with Epstein-barr virus-negative carcinoma. Clin Cancer Res 10: 1698-1705, 2004.

33. zur Hausen A, van Grieken NC, Meijer GA, et al: Distinct chromosomal aberrations in Epstein-Barr virus-carrying gastric carcinomas tested by comparative genomic hybridization. Gastroenterology 121: 612-618, 2001.

34. Chang MS, Uozaki H, Chong JM, et al: $\mathrm{CpG}$ island methylation status in gastric carcinoma with and without infection of Epstein-Barr virus. Clin Cancer Res 12: 2995-3002, 2006.

35. Chong JM, Sakuma K, Sudo M, et al: Global and non-random $\mathrm{CpG}$-island methylation in gastric carcinoma associated with Epstein-Barr virus. Cancer Sci 94: 76-80, 2003.

36. Hino R, Uozaki H, Murakami N, et al: Activation of DNA methyltransferase 1 by EBV latent membrane protein $2 \mathrm{~A}$ leads to promoter hypermethylation of PTEN gene in gastric carcinoma. Cancer Res 69: 2766-2774, 2009.

37. Fu DX, Tanhehco Y, Chen J, et al: Bortezomib-induced enzymetargeted radiation therapy in herpesvirus-associated tumors. Nat Med 14: 1118-1122, 2008

38. Jung EJ, Lee YM, Lee BL, Chang MS and Kim WH: Lytic induction and apoptosis of Epstein-Barr virus-associated gastric cancer cell line with epigenetic modifiers and ganciclovir. Cancer Lett 247: 77-83, 2007.

39. Hsu JT, Chen TC, Tseng JH, et al: Impact of HER-2 overexpression/ amplification on the prognosis of gastric cancer patients undergoing resection: a single-center study of 1,036 patients. Oncologist 16: 1706-1713, 2011. 